\title{
Temporary tarsorrhaphy in the rat
}

Temporarily suturing the eyelids together may be necessary in certain studies where corneal dryness or ulceration may appear as secondary complications to experimental manipulation. This column describes the surgical technique of tarsorrhaphy in general, and details its use in the rat.

Tarsorrhaphy, or blepharorrhaphy, refers to a surgical procedure in which the upper and lower eyelids are sutured together for the purpose of shortening or entirely closing the palpebral fissure (the opening between the eyelids) ${ }^{1}$. The procedure is classified as a partial or a complete tarsorrhaphy depending on whether all or only a portion of the palpebral fissure is occluded. Tarsorrhaphy is further characterized as either temporary or permanent.

\section{INDICATIONS}

Temporary tarsorrhaphy may be used following ophthalmic surgery to immobilize the eyelids, thereby preventing wound contracture during the healing process ${ }^{2}$, or to protect the cornea following trauma or proptosis of the globe ${ }^{3}$. Temporary tarsorrhaphy has been shown to aid the healing of corneal epithelial defects ${ }^{4}$. Specific situations encountered in animals that may warrant the use of this procedure include the following:

- After plastic surgery, temporary tarsorrhaphy may be used to immobilize the eyelids and keep them splinted during healing. Common plastic surgeries performed in canines, for instance, include corrective entropion, ectropion, lacerations, and mass removal.

- In cases of spontaneous or experimentally induced corneal pathology (neuroparalytic or neurotropic keratitis), the procedure may be used to protect the cornea.

- In laboratory experiments in which the ophthalmic nerve of rats serves as a model for spinal cord injury and regeneration, temporary tarsorrhaphy can minimize complications associated with facial paralysis.
- Premature opening of the palpebral fissure is an infrequent indication for temporary tarsorrhaphy in puppies ${ }^{5}$.

\section{PREPARATION}

An animal should be under general anesthesia for the tarsorrhaphy procedure because it is unlikely to remain still with the use of topical anesthetic alone. A sterile ophthalmic ointment must be placed onto the cornea for protection. A dilute betadine solution or other non-irritating preparation should be used to gently scrub the eyelids, followed by a saline rinse to remove any residual scrub. Aseptic technique, including the use of a small sterile drape, should be used to prepare the patient and for the duration of the procedure ${ }^{6}$. Lateral recumbency is the best position for surgery, although some surgeons prefer ventral recumbency to minimize the time required to reposition and prepare the opposite eye.

\section{EQUIPMENT}

Atraumatic microsurgical forceps should be used to grasp the eyelids while placing sutures. Small needle holders capable of grasping 5.0-6.0 suture material (such as synthetic polydioxanone (PDS), or nonabsorbable silk) are necessary. Stents or small hollow tubes used to decrease the tension of the suture material can be fashioned out of 0.5 -cm-long pieces of plastic, Teflon, or rubber. The rubber tubing from winged infusion sets or cut pieces of the Teflon portion of an intravenous catheter work well as small stents. Magnification using either a surgical microscope or a magnification head loupe is useful in small animals.

\section{PROCEDURE}

In temporary tarsorrhaphy, marginal eyelid adhesions are not necessary (as they are in permanent tarsorrhaphy). The lids may be held in apposition with sutures alone, provided the suture is run through a stent to distribute the tension and avoid pressureinduced necrosis of the eyelids.

Mattress sutures are placed in the eyelids so that the needle emerges through the central aspect of the eyelid margin ${ }^{2}$. All sutures should be placed equidistant from the eyelid margin to maintain symmetry. The size of the animal's globe will dictate how many sutures are necessary. In a 200-300 g rat only one or two sutures are required.

First, place the suture through a stent (S1) and then through the anterior (epidermal)

\section{EOUIPMENT FOR TEMPORARY TARSORRHAPHY}

Microsurgical forceps

Needle holders

Fine suture material (e.g., synthetic polydioxanone or nonabsorbable silk)

Stents (plastic/rubber tubing/Teflon catheters)

Dilute betadine solution or non-irritating preparation

Saline flush

Ocular lubricant

Magnification equipment (e.g., operating microscope or head loupes)

Fluids for subcutaneous administration

Pain medication

Surgical gloves 


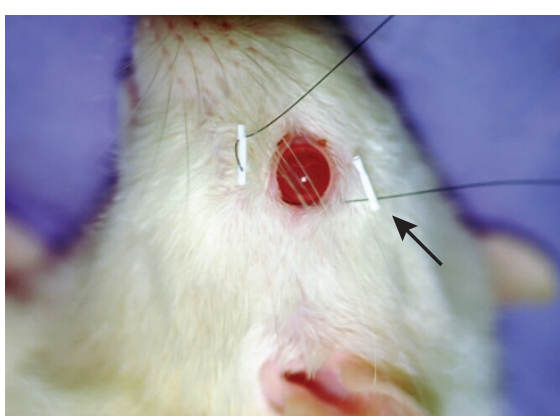

FIGURE 1 | A temporary tarsorrhaphy is performed by placing a 6.0 PDS suture through a Teflon stent (S1) (see arrow) and then through the anterior aspect of the upper eyelid, exiting through the middle aspect of the upper eyelid margin. The suture is then passed through the middle of lower eyelid margin, traveling subcutaneously and exiting from the anterior aspect of the lower eyelid. Then the suture is run through a second stent (S2) and placed back through the same stent (S2) again, in preparation for needle placement through the anterior aspect of the lower eyelid.

portion of the eyelid, running it subcuticular to the central aspect of the eyelid margin without completely penetrating the eyelid. Next, put the suture through the central aspect of the opposite eyelid margin so that it travels subcuticular and exits from the anterior eyelid before moving through a second stent (S2). Then bring the suture material through that same stent (S2) (Fig. 1) and through the anterior portion of the eyelid (again traveling subcuticular), exiting at the middle portion of the eyelid margin.

Next, place the suture through the middle aspect of the opposite eyelid margin, exiting at the anterior portion of the eyelid. Make the final pass through the initial stent (S1) (Fig. 2). Lastly, tie the suture, applying just enough pressure to gently appose the eyelids (Fig. 3). If necessary, sutures can be placed such that the medial canthus is left open for the administration of ophthalmic antibacterial ointment, ophthalmic lubricating ointment, or drops. Topical ophthalmic medicators are used, even with a tarsorrhaphy in place, since they are able to penetrate the eyelids to the eyes below.

\section{POSTOPERATIVE CONSIDERATIONS}

Warm compresses can be applied for 24 hours after surgery to minimize swelling and to help relieve local discomfort; systemic analgesics should be administered as needed. Tarsorrhaphy sutures can be removed 10-28 days after surgery or sooner if persistent pain, mucopurulent exudate, malaise, or fever develop ${ }^{3}$. The duration of suture placement depends on the condition of the eye and resolution of predisposing factors.

Occasionally patients may require a restraint collar to prevent them from dislodging the sutures ${ }^{7,8}$. Animal caretakers should be sure food and water are easily accessible. It is important to offer the animal food by hand and to monitor its body weight daily. Fluid supplementation is recommended for the initial 24 hours after the procedure.

\section{Complications}

It is important that the sutures travel subcuticular and not penetrate completely through the eyelid into direct contact with the cornea (i.e., this should not be a full thickness suture) or it can result in chronic irritation and ulceration. If ocular problems persist despite tarsorrhaphy and administration of topical ophthalmic medications, it is critical to re-evaluate the eye. Depending on suture placement in the eyelid, if the sutures are tied too tight, the eyelid margins may invert or evert, resulting in further discomfort for the patient. Be sure to tie the suture only as tight as necessary to appose the eyelids.

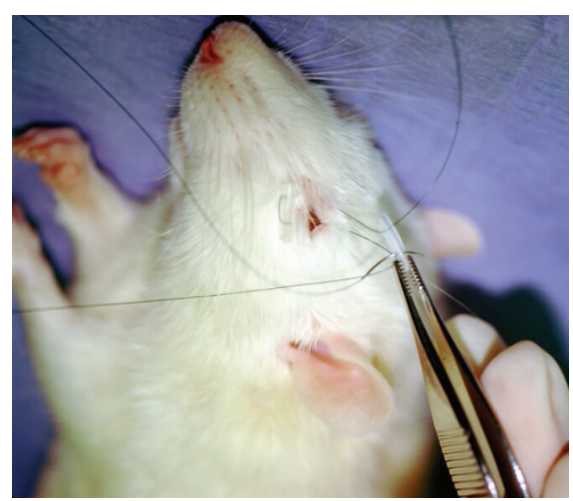

FIGURE 2 | Placement of suture material through the final stent (S2), created from a fragment of a winged infusion set.

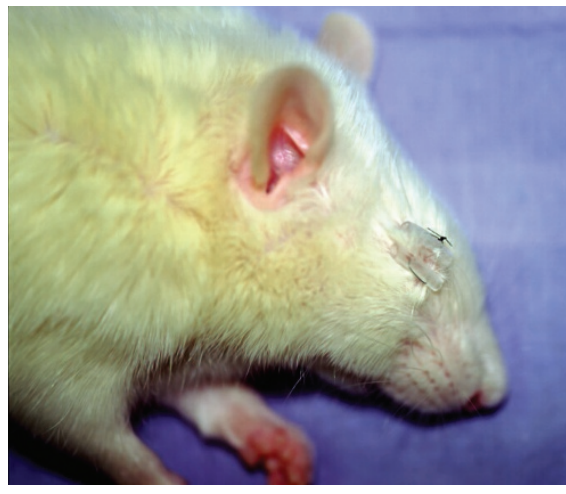

FIGURE 3 | An example of the final temporary tarsorrhaphy. The knot is tied on the outer aspect of a single, perpendicular mattress suture using (winged infusion set) tubing stents.

\section{ALTERNATIVE TREATMENT}

The use of botulinum toxin to produce drooping of the upper eyelid has been reported as an alternative to temporary tarsorrhaphy. This involves an injection into the upper eyelid to temporarily paralyze the superior tarsal muscle (known as the levator palpebrae muscle in humans) that acts to hold the upper lid in an elevated position. It usually takes effect after a few days and lasts for 6-8 weeks ${ }^{9}$.

1. Dorland's Illustrated Medical Dictionary $27^{\text {th }}$ edn (W.B.Saunders, Philadelphia, 1988).

2. Hedlund, C.S. in Small Animal Surgery 2nd edn. (eds. Fossum, T.W. et al.) 216-225 (Mosby, St. Louis, 2002).

3. Bistner, S.I., Aguirre, G. \& Batik, G. (eds.) Atlas of Veterinary Ophthalmalic Surgery 46-49 (W.B. Saunders, Philadelphia, 1977).

4. Kitchens, J., Kinder, J. \& Oetting, T. The drawstring temporary tarsorrhaphy technique. Arch. Ophthalmol. 120(2), 187-190 (2002).

5. Gelatt, K.N. in Essentials of Veterinary Ophthalmology (eds. Katz, S., Battaglia, D. \& Williams, P.C.) Ch. 3 (Lippincott Williams and Wilkins, Baltimore, 2000).

6. Kunimoto, D.Y., Kanitkar, K.D., Makar, M.S., Friedberg, M.A. \& Rapuano, C.J. (eds.) Wills Eye Manual: Office and Emergency Room Diagnosis \& Treatment of Eye Disease 4th edn. Ch. 4 (Lippincott Williams \& Wilkins, Philadelphia, 2004).

7. Brown, C. Restraint collars. Part I: Elizabethan collars and other types of restraint collars. Lab Anim. (NY) 35(2), 23-25 (2006).

8. Brown, C. Restraint collars. Part II: specific issues with restraint collars. Lab Anim. (NY) 35(3), 25-27 (2006).

9. Lee, V., Currie, Z. \& Collin, J.R. Ophthalmic management of facial nerve palsy. Eye 18(12), 1225-1234 (2004). 